$11-1-2000$

\title{
Paramagnetic Meissner Effect in Nb Disks
}

Petru S. Fodor

Cleveland State University, p.fodor@csuohio.edu

Lowell E. Wenger

Wayne State University, wenger@physics.wayne.edu

Follow this and additional works at: https://engagedscholarship.csuohio.edu/sciphysics_facpub

Part of the Physics Commons

How does access to this work benefit you? Let us know!

\section{Publisher's Statement}

NOTICE: this is the author's version of a work that was accepted for publication in Physica C:

Superconductivity. Changes resulting from the publishing process, such as peer review, editing, corrections, structural formatting, and other quality control mechanisms may not be reflected in this document. Changes may have been made to this work since it was submitted for publication. A definitive version was subsequently published in Physica C: Superconductivity, 341, 3, November 2000 DOI\#10.1016/S0921-4534(00)00991-6

\section{Repository Citation}

Fodor, Petru S. and Wenger, Lowell E., "Paramagnetic Meissner Effect in Nb Disks" (2000). Physics Faculty Publications. 205.

https://engagedscholarship.csuohio.edu/sciphysics_facpub/205

This Article is brought to you for free and open access by the Physics Department at EngagedScholarship@CSU. It has been accepted for inclusion in Physics Faculty Publications by an authorized administrator of EngagedScholarship@CSU. For more information, please contact library.es@csuohio.edu. 


\title{
Paramagnetic Meissner effect in $\mathrm{Nb}$ disks
}

\author{
Petru S. Fodor and L.E. Wenger
}

Further details of the zero-field-cooled-magnetization and field-cooled-magnetization results on $\mathrm{Nb}$ disks exhibiting the paramagnetic Meissner effect (PME) are described. These studies indicate that two well-defined temperatures can be associated with features in the magnetization results. The higher characteristic temperature $T_{u}$ is correlated with appearance of the paramagnetic moment and is strongly dependent upon the process used in forming the diskshaped geometry of these $\mathrm{Nb}$ samples. The lower temperature $\mathrm{T}_{\mathrm{p}}$ is associated with the "intrinsic coupling" of the interior $\mathrm{Nb}$ platelets. These latest results are shown to be consistent with the flux compression model for the appearance of the PME.

\section{INTRODUCTION}

The paramagnetic Meissner effect (PME) is characterized by a positive field-cooled magnetization below the superconducting transition temperature $T_{c}$. Although this behavior has been suggested as a possible manifestation of $d$-wave superconductivity in the cuprates [1], our previously reported observation of a PME on macroscopic $\mathrm{Nb}$ disks [2] for fields normal to the disks required an alternative explanation. One suggestion was that the positive moment arose from a compression of the magnetic flux [3] into the sample due to inhomogeneous cooling. In order to further clarify the nature of the PME in these macroscopic disks, additional details of our magnetization results on $0.127-\mathrm{mm}$ thick, 6.0-mm diameter $\mathrm{Nb}$ disks will be presented.

\section{RESULTS}

Figure 1 shows detailed zero-field-cooled magnetization (ZFCM) and field-cooled magnetization (FCM) data for field orientations, both parallel and perpendicular to the $\mathrm{Nb}$ disk. The dashed vertical lines indicate the temperatures $T_{u}$ and $T_{p}$ where $T_{u}$ is the temperature that the positive moment first appears and $T_{p}$ is the temperature that the positive contribution stops increasing in magnitude during cooling. As reported previously [4], the FCM behavior for the perpendicular orientation consists of two contributions: a hysteretic behavior arising from the flux trapping and diamagnetic screening associated with a typical type-II bulk superconducting sample, and a nearly reversible behavior associated with the PME which appears and disappears at $T_{u}$. At low-fields, the abrupt appearance of this positive moment upon cooling below $\mathrm{T}_{\mathrm{u}}$ appears to be fairly spontaneous, similar to the onset of global diamagnetic screening currents in a homogeneous bulk superconductor rather than the viscous nature exemplified by flux flow.

It is also evident that these temperatures can be identified with certain characteristic features in the ZFCM data. $T_{p}$ can be associated with the temperature where the diamagnetic response is a maximum and full flux exclusion occurs, while $T_{u}$ is the temperature where global diamagnetic screening disappears during warming. Also the two tier-like behaviors in the ZFCM 


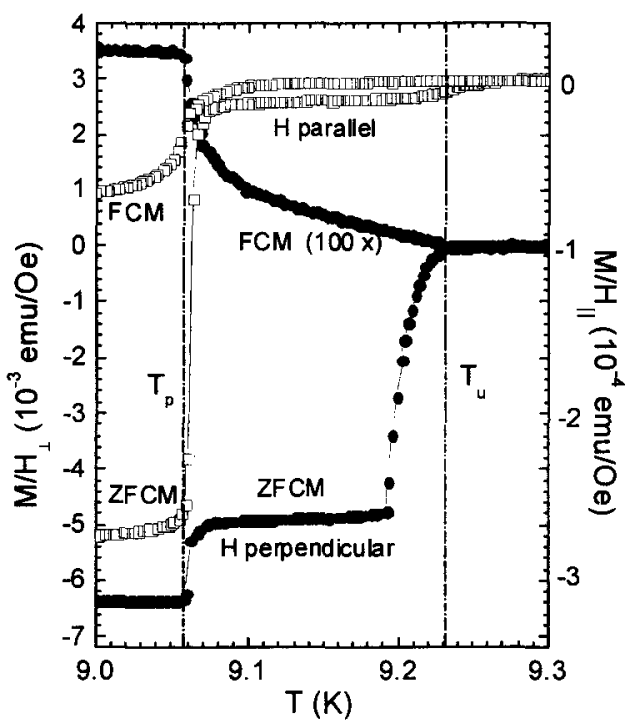

Figure 1. ZFCM/H and FCM/H for $\mathrm{H}$ parallel and perpendicular to the $\mathrm{Nb}$ disk.

results for both field directions would be indicative of a "two-phase" material. Since the demagnetization field effect is negligible in the parallel orientation, the ZFCM parallel-field results indicate nearly full flux exclusion $(95 \%$ of $V / 4 \pi)$ for $T<T_{p}$ while less than $5 \%$ of the disk contributes to the diamagnetic response between $T_{p}$ and $T_{u}$. However previous studies on abraded disks indicate that full flux exclusion can approach temperatures of $9.26 \mathrm{~K}$, the superconducting transition temperature of the individual $\mathrm{Nb}$ platelets forming the disk. Thus we speculate that the temperatures $T_{p}$ and $T_{u}$ are related to the strength of the superconducting coupling between the platelets with $T_{p}$ being characteristic of the intrinsic coupling between platelets that arises from the cold-rolling process of the $\mathrm{Nb}$ sheet and $\mathrm{T}_{\mathrm{u}}$ with the coupling of a smaller number of platelets that results from the cutting process of the disk itself. In fact, $T_{u}$ is found experimentally to vary from disk to disk and is very dependent on the cutting/punching process. Furthermore, the observation that the ZFCM perpendicular to the field only changes by about $20 \%$ for temperatures above and below $T_{p}$ suggests that the outer rim of the disk is the location of the stronger coupling since the ZFCM magnitude is essentially only dependent upon the radius $\left(\propto \mathbf{r}^{3}\right)$ and not the thickness of the disk. Thus the disk can be thought to be composed of micron-sized platelets with a $\mathrm{T}_{\mathrm{c}}=9.26 \mathrm{~K}$ that are cold-pressed together with a granular coupling occurring at $9.06 \mathrm{~K}$. However during the cutting/punching of the disk, the disk's rim is compressed with slightly greater pressure and a correspondingly higher coupling temperature $T_{u}$ results. This two tier-like structure in the ZFCM has been independently observed by compressing another $\mathrm{Nb}$ disk with two cylinders so that a rim of approximately 0.03 $\mathrm{mm}$ in size has a greater coupling. Moreover the abrupt changes in the linear M-vs-H curves for this $\mathrm{Nb}$ disk in the temperature range $T_{p}<T<T_{u}$ are consistent with $M-v s-H$ curves measured for a ring-like structure.

Thus, the formation of a positive moment in these macroscopic $\mathrm{Nb}$ disks would be most consistent with the flux compression model. The magnetic flux would be trapped by the outer disk region with a higher "transition" temperature and then would be compressed with decreasing temperature as the inner regions become coupled. The relative small magnitude of the positive moment as compared to $V / 4 \pi$ would indicate that the compression occurs only within a small distance from the disk's edge.

\section{REFERENCES}

1. T.M. Rice and M. Sigist, Rev. Mod. Phys. 67 (1995) 503.

2. D.J. Thompson, et al., Phys. Rev. Lett. 75 (1995) 529.

3. A.E. Koshelev and A.I. Larkin, Phys. Rev. B 52 (1995) 13559.

4. L. Pust, et al., Phys. Rev. B 58 (1998) 14191. 